\title{
Erratum to: The inhibitory effect of chimeric decoy oligodeoxynucleotide against NF- $\kappa$ B and Sp1 in renal interstitial fibrosis
}

\author{
Kyung-Hyun Kim • Ji-Hyun Park • Woo-Ram Lee • \\ Jae-Shin Park • Hyun-Chul Kim • Kwan-Kyu Park
}

Published online: 23 April 2013

(C) Springer-Verlag Berlin Heidelberg 2013

\section{Erratum to: J Mol Med \\ DOI 10.1007/s00109-012-0972-2}

It has been brought to our attention as authors that the right panel in Fig. 3a, showing the immunofluorescence staining for Chi decoy ODN after 7 days, was published incorrectly. Specifically, it was mistakenly duplicated from Fig. 6b. The right panel in Fig. 3a has been replaced with the true immunofluorescence image and the corrected Fig. 3 is given below. The authors claim that this correction does not influence the conclusion of the study and would like to apologize for this error.

The online version of the original article can be found at http://dx.doi.org/ 10.1007/s00109-012-0972-2.

K.-H. Kim · J.-H. Park • W.-R. Lee · K.-K. Park $(\bowtie)$

Department of Pathology, College of Medicine, Catholic

University of Daegu, 3056-6 Daemyung 4-dong,

Daegu, Nam-gu 705-718, Republic of Korea

e-mail:kkpark@cu.ac.kr

J.-S. Park

Department of Urology, College of Medicine, Catholic University of Daegu, 3056-6 Daemyung 4-dong,

Daegu, Nam-gu 705-718, Republic of Korea

H.-C. Kim

Department of Internal Medicine, Division of Nephrology, School

of Medicine, Keimyung University, 194 Dongsan-dong,

Daegu, Jung-gu 700-712, Republic of Korea 
A
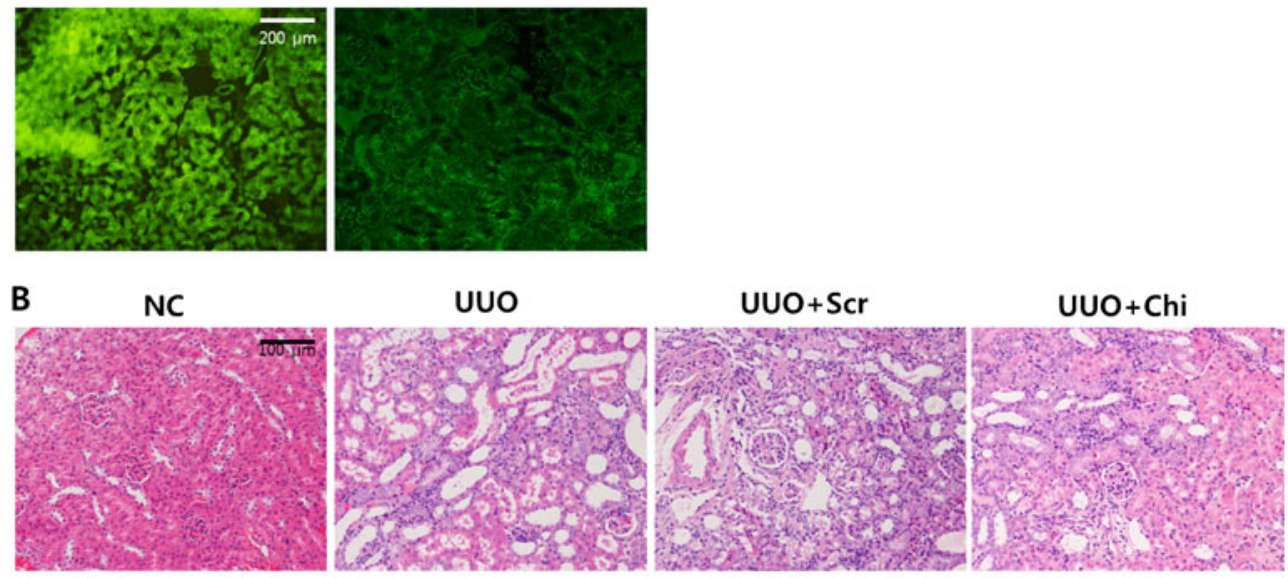

C
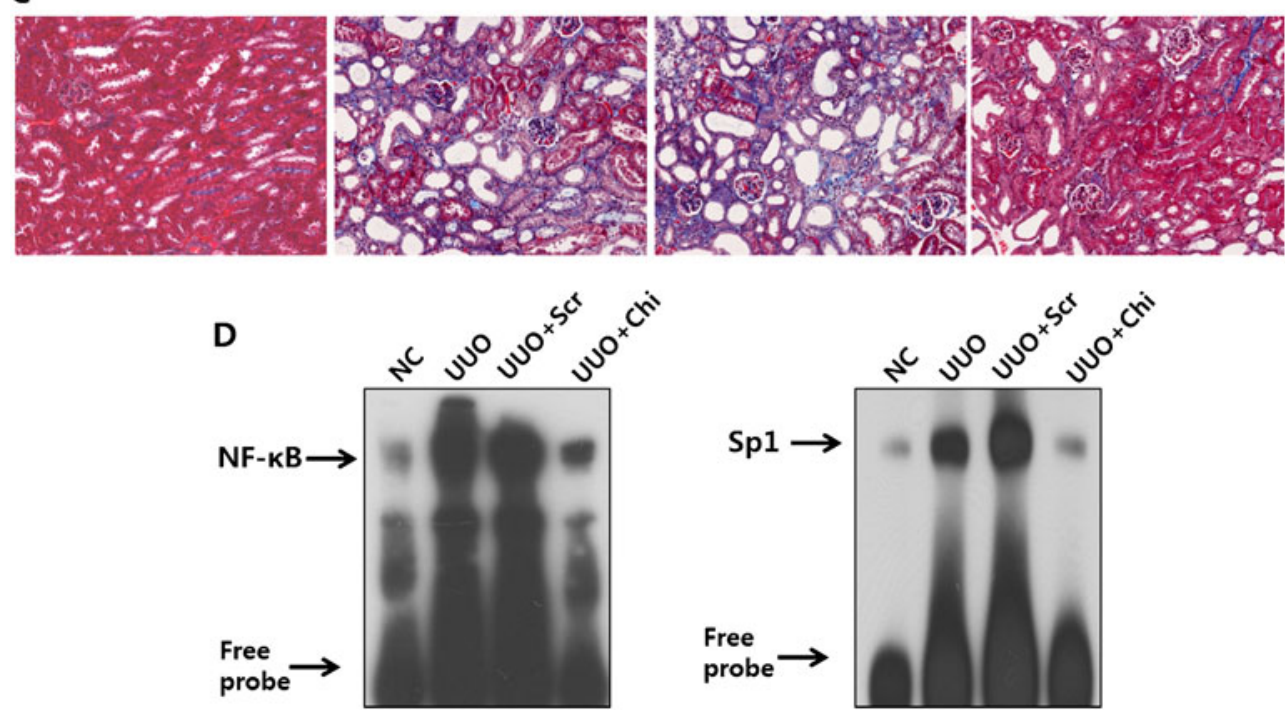